\title{
Fusarium graminearum PKS14 is involved in orsellinic acid and orcinol synthesis
}

Jørgensen, Simon Hartung ; Frandsen, Rasmus John Normand; Nielsen, Kristian Fog; Lysøe, Erik; Sondergaard, Teis Esben; Wimmer, Reinhard; Giese, Henriette; Sørensen, Jens Laurids

\section{Published in:}

Fungal Genetics and Biology

Link to article, DOI:

10.1016/j.fgb.2014.06.008

Publication date:

2014

Document Version

Publisher's PDF, also known as Version of record

Link back to DTU Orbit

Citation (APA):

Jørgensen, S. H., Frandsen, R. J. N., Nielsen, K. F., Lysøe, E., Sondergaard, T. E., Wimmer, R., Giese, H., \& Sørensen, J. L. (2014). Fusarium graminearum PKS14 is involved in orsellinic acid and orcinol synthesis. Fungal Genetics and Biology, 70, 24-31. https://doi.org/10.1016/j.fgb.2014.06.008

\section{General rights}

Copyright and moral rights for the publications made accessible in the public portal are retained by the authors and/or other copyright owners and it is a condition of accessing publications that users recognise and abide by the legal requirements associated with these rights.

- Users may download and print one copy of any publication from the public portal for the purpose of private study or research.

- You may not further distribute the material or use it for any profit-making activity or commercial gain

- You may freely distribute the URL identifying the publication in the public portal 


\title{
Fusarium graminearum PKS14 is involved in orsellinic acid and orcinol synthesis
}

\author{
Simon Hartung Jørgensen ${ }^{a}$, Rasmus John Norman Frandsen ${ }^{b}$, Kristian Fog Nielsen ${ }^{\mathrm{b}}$, Erik Lysøe ${ }^{\mathrm{c}}$, \\ Teis Esben Sondergaard ${ }^{a}$, Reinhard Wimmer ${ }^{a}$, Henriette Giese ${ }^{a}$, Jens Laurids Sørensen ${ }^{\mathrm{a}, *}$ \\ a Department of Biotechnology, Chemistry and Environmental Engineering, Aalborg University, Sohngaardsholmsvej 49, DK-9000 Aalborg, Denmark \\ ${ }^{\mathrm{b}}$ Department of Systems Biology, Technical University of Denmark, Søltofts Plads, DK-2800 Kgs. Lyngby, Denmark \\ ${ }^{\mathrm{c}}$ Department of Plant Health and Plant Protection, Bioforsk-Norwegian Institute of Agricultural and Environmental Research, 1432 Ås, Norway
}

\section{A R T I C L E I N F O}

\section{Article history:}

Received 6 December 2013

Accepted 19 June 2014

Available online 8 July 2014

\section{Keywords:}

Secondary metabolites

Polyketide synthase 14

Overexpression

Regulation

Fusarium

\begin{abstract}
A B S T R A C T
The available genome sequences show that the number of secondary metabolite genes in filamentous fungi vastly exceeds the number of known products. This is also true for the global plant pathogenic fungus Fusarium graminearum, which contains 15 polyketide synthase (PKS) genes, of which only 6 have been linked to products. To help remedy this, we focused on PKS14, which has only been shown to be expressed during plant infections or when cultivated on rice or corn meal (RM) based media. To enhance the production of the resulting product we introduced a constitutive promoter in front of PKS14 and cultivated two of the resulting mutants on RM medium. This led to the production of two compounds, which were only detected in the PKS14 overexpressing mutants and not in the wild type or PKS14 deletion mutants. The two compounds were tentatively identified as orsellinic acid and orcinol by comparing spectroscopic data (mass spectroscopy and chromatography) to authentic standards. NMR analysis of putative orcinol isolated from the PKS14 overexpressing mutant supported our identification. Orcinol and orsellinic acid, not previously detected in Fusarium, have primarily been detected in lichen fungi. Orsellinic acid is hypothesized to be the PKS release product which is transformed to orcinol through decarboxylation. Phylogenetic analyses of PKSs placed PKS14 in a subclade of known OA synthases. Expression analysis by microarray of 55 experiments identified seven genes near PKS14 that were expressed in a similar manner. One of the seven genes encodes a predicted carboxylase, which could be responsible for transforming orsellinic acid to orcinol.
\end{abstract}

(c) 2014 Elsevier Inc. All rights reserved.

\section{Introduction}

The filamentous fungus Fusarium graminearum has the genetic potential of producing a wide array of secondary metabolites (Hansen et al., 2012b). Whole-genomic analysis have identified 15 polyketide synthases (PKS1-15) (Kroken et al., 2003; Gaffoor and Trail, 2006), where six have been linked to metabolites: PKS3 - perithecial pigment (Gaffoor et al., 2005), PKS4/13 - zearalenone (Kim et al., 2005; Gaffoor and Trail, 2006), PKS9 - fusarielins (Sørensen et al., 2012), PKS10 - fusarin C (Gaffoor et al., 2005) and PKS12 - aurofusarin (Frandsen et al., 2006). The remaining PKSs have not yet been assigned products, even though they are expressed under tested conditions (Gaffoor et al., 2005). As $F$. graminearum infects cereal grains, there is a risk that these unidentified metabolites can contaminate our food sources

\footnotetext{
* Corresponding author. Fax: +45 98141808.

E-mail address: jls@bio.aau.dk (J.L. Sørensen).
}

(Burlakoti et al., 2008), which emphasizes the need to determine the full metabolic capacity of $F$. graminearum.

The genes involved in the syntheses of polyketides are often found in gene clusters encoding the synthase and tailoring enzymes, transporters and local cluster activating transcription factor(s) (Keller and Hohn, 1997). Gene clusters, which have not been linked to secondary metabolites, can be activated by many different approaches including changing the cultivation practice according to the OSMAC (one strain, many compounds) method, where light, air, nutrition sources and $\mathrm{pH}$ are varied (Bode et al., 2002) or co-cultivation with other competing microorganism (Schroeckh et al., 2009). Secondary metabolic pathways have also been induced through chromatin modification using chemical agents or manipulation of chromatin modifying proteins like LaeA (Fisch et al., 2009) or by the overexpression of any transcription factor encoding genes located proximal to the PKSs (Bergmann et al., 2007). The low success rate of these strategies is illustrated by our efforts to over express putative transcription factors near 
PKS3, 5, 7, 9, 11 and 15 gene clusters in $F$. graminearum, which was only successful in the case of PKS9 leading to the production of novel fusarielins (Sørensen et al., 2012). In Aspergillus nidulans overexpression of transcription factors near 17 secondary metabolite gene clusters led to induction of only five products (Ahuja et al., 2012). However, when Ahuja et al. (2012) used overexpression of the PKS genes directly, they managed to identify the release products of eight non-reducing (NR)-PKSs of which downstream metabolites were known or identified for six.

PKS14 is the only NR-PKS with a claisen-type cyclase (CLC) domain in $F$. graminearum that has not been linked to synthesis of a chemical product (Hansen et al., 2012b). CLC domains are responsible for releasing the product from the synthase and the presence of this domain suggests that PKS14 is not dependent on other enzymes for releasing its product. The gene appears to be induced by plant stimuli as it is transcribed under wheat and barley infection (Lysøe et al., 2011) and when F. graminearum is cultivated on rice or corn media (Gaffoor et al., 2005). Gaffoor et al. (2005) deleted PKS14, which resulted in reduced growth. However, no change in metabolite profile was detected in the mutant compared to the wild-type. As there is no transcription factor in the proximity of PKS14, we overexpressed the PKS14 gene itself using the same strategy as Ahuja et al. (2012), which led to production of orsellinic acid and orcinol.

\section{Materials and methods}

\subsection{Phylogenetic analyses}

Amino acid sequences of KS domains from selected NR-PKSs were retrieved from the Fusarium comparative database (http:// www.broad.mit.edu/annotation/fungi/fusarium), the Aspergillus comparative database (www.broadinstitute.org/annotation/genome/aspergillus_group) and GenBank (http://www.ncbi.nlm.nih. gov). The PKS14 PT domain was identified by BlastP analyses using the PT domain of PKSA from Aspergillus parasiticus as previously described by Li et al. (2010). The PKS14 PT domain was then used to identify PT domains in the remaining NR-PKSs. The sequences were aligned by clustalW and a phylogeny tree was constructed by the neighbor joining (NJ) algorithm with 1000 bootstrap replicates in the Molecular Evolutionary Genetics Analysis software (MEGA 5.2) (Tamura et al., 2011).

\subsection{Expression analyses and cluster prediction}

Hierarchical clustering was performed in CLC Genomics Workbench 6.5.1, using 175 microarray samples from 55 experiments; a combination of published results found in the plant expression database (plexdb.com) and unpublished results. The variable conditions in the experiments include plant infection, sexual - and asexual development as well as different media with varying nitrogen sources. The Affymetrix CEL files were processed through the "affy" package in Bioconductor and normalized using the quantile method in CLC Genomics Workbench. The hierarchical clustering of features was performed with single linkage using manhattan distance. The function of the proteins encoded by the cluster genes was predicted based on comparison to proteins in the NCBI Conserved Domain Database (Marchler-Bauer et al., 2011).

\subsection{Construction of PKS14 overexpression and deletion mutants}

The flanking regions of PKS14 was PCR amplified from F. graminearum PH-1 using primers PKS14-KO1-PKS14-KO4 listed in Supplementary Table 1 and Pfu polymerase (Stratagene, La Jolla, CA, USA). The amplified fragments were introduced into the
pRF-HU2 vector flanking the hygromycin B resistance cassette by USER cloning (Frandsen et al., 2008). Overexpression of PKS14 was achieved by introducing the constitutive promoter gpdA from A. nidulans in front of PKS14 using a similar approach as described previously. A fragment upstream of PKS14 and a fragment from the beginning of the gene were amplified by $\mathrm{PCR}$, using primers PKS14-01-PKS14-04, and cloned into the linearized pRF-HU2E vector containing the gpdA promoter. Transformation of F. graminearum was carried out by Agrobacterium tumefaciens mediated transformation as described previously (Malz et al., 2005) and the resulting mutants were initially verified by diagnostic PCR using a forward primer annealing to gDNA outside the border region and a reverse primer annealing to the hygromycin resistance gene. The number of inserts was determined by Southern blotting (Hansen et al., 2012a). Genomic DNA was extracted from the transformed fungi and subjected to a restriction digestion using HindIII fast digest (Fermentas, Thermo Fisher Scientific, Inc., Denmark) followed by hybridization with probe of $588 \mathrm{bp}$ corresponding to a part of the hygromycin resistance gene.

\subsection{Transcription analyses}

Extraction of RNA from $F$. graminearum was adapted from the method used by Droce et al. (2013). In brief, F. graminearum was cultivated for 9 days on solid rice meal (RM) medium ( $75 \mathrm{~g} / \mathrm{L}$ rice meal, $20 \mathrm{~g} / \mathrm{L}$ agar and $1 \mathrm{~mL} / \mathrm{L}$ trace solution $\left(1.0 \mathrm{~g} \mathrm{ZnSO}_{4}\right.$ and $0.5 \mathrm{~g}$ $\mathrm{CuSO}_{4}$ in $100 \mathrm{~mL} \mathrm{H}_{2} \mathrm{O}$ ) pH 6.7). Total RNA was extracted with RNeasy plant mini-kit (Qiagen, Hilden, Germany) following the recommended protocol including the QIAshredder column (Qiagen). First-stranded cDNA was synthesized using poly(dT) primer (15 bp) and Superscript III reverse transcriptase (Invitrogen Life Technologies, Carlsbad, CA, USA) according to the manufacturer including the RNaseOUT step. Expression of genes in the PKS14 cluster was determined by PCR using the primers listed in the Supplementary Table 1 and paq5000 DNA polymerase (Stratagene). The amplified region included a predicted intron which would result in a $60 \mathrm{bp}$ between gDNA and cDNA. Translation elongation factor $1 \alpha$ (TEF-1 $\alpha$ ) and $\beta$-tubulin were included as controls.

\subsection{Extraction and purification of secondary metabolites}

F. graminearum was grown on a solid RM medium in petri dishes for two weeks at $25^{\circ} \mathrm{C}$. The microscale extraction of metabolites was adapted from (Smedsgaard, 1997). Nine agar plugs were sonicated for $45 \mathrm{~min}$ with $1 \mathrm{~mL}$ ethyl acetate:dichloromethane:methanol (3:2:1) buffered with $1 \%$ formic acid. The extract were lyophilized and dissolved ultrasonically in $600 \mu \mathrm{L}$ methanol.

The samples were initially analyzed on an Agilent 1260 LC system (Agilent Technologies, Waldbronn, Germany) equipped with a diode array detector. Five $\mu \mathrm{L}$ sample were injected and separated on a $100 \times 2.1 \mathrm{~mm}$ kinetex $2.6 \mu \mathrm{m}$ Hexyl-Phenyl (Phenomenex, Torrance, CA, USA) using a flow of $0.400 \mathrm{~mL} / \mathrm{min}$ with a linear water-acetonitrile gradient, where both eluents were buffered with 50 ppm trifluoroacetic acid. The gradient started at $15 \%$ acetonitrile and reached $100 \%$ acetonitrile in $20 \mathrm{~min}$, which were held for $5 \mathrm{~min}$. The extracts were subsequently analyzed by ultrahigh-performance liquid chromatography combined with high resolution mass spectrometry (UHPLC-HRMS) on an Agilent 1290 UHPLC system (Agilent technologies, Santa Clara, CA, USA) equipped with a $25 \mathrm{~cm}, 2 \mathrm{~mm}$ ID, $2.6 \mu \mathrm{m}$ Agilent Poroshell phenyl hexyl column, and coupled to an Agilent 6550 quadrupole Time of Flight (qTOF) high resolution mass spectrometer equipped with an electrospray source. The qTOF was operated at $2 \mathrm{GHz}$ in the extended dynamic range mode at a resolution of 30,000 FMWH. Subsamples of $1 \mu \mathrm{L}$ were separated at $60^{\circ} \mathrm{C}$ with a flow rate of $0.35 \mathrm{~mL} / \mathrm{min}$ using a linear water-acetonitrile system both 
containing $20 \mathrm{mM}$ formic acid. The acetonitrile gradient started from $10 \%$ and was raised to $100 \%$ in $15 \mathrm{~min}$, and held for $3 \mathrm{~min}$ prior to returning to the start conditions. The qTOF was operated in positive mode making full scans from $\mathrm{m} / z 100$ to 170010 times per sec. Furthermore, MS/MS spectra was collected at 10, 20, and $40 \mathrm{eV}(\mathrm{m} / \mathrm{z} 30$ $1700)$ from all major chromatographic peaks with mass in the range $m / z$ 120-1700 (Nielsen et al., 2013). UV/Vis data and the full scan MS data were compared to the data listed in Nielsen et al. (2011).

For structure elucidation large scale extraction and purification was carried out. Metabolites were extracted from 37 RM plates of two weeks old cultures by ultrasonic treatment for $45 \mathrm{~min}$ in ethyl acetate buffered with $1 \%$ formic acid. The extract was evaporated to dryness on a rotary evaporator, resuspended in $20 \mathrm{~mL}$ methanol and the metabolites were then fractionated on a Strata C18-E ( $55 \mu \mathrm{m}, 70 \AA$ ), $50 \mathrm{~g} / 150 \mathrm{~mL}$, Giga Tube (Phenomenex, Torrance, CA, USA). The extract were loaded on the column in a $10 \%$ methanol solution, washed with $200 \mathrm{~mL} 20 \%$ methanol and eluted with $200 \mathrm{~mL} \mathrm{40 \%} \mathrm{acetonitrile.} \mathrm{The} \mathrm{final} \mathrm{purification} \mathrm{was} \mathrm{performed} \mathrm{by}$ multiple cycles on an Agilent 1260 semi-preparative HPLC system equipped with a $150 \times 10 \mathrm{~mm}$ Gemini $5 \mu \mathrm{m}$ C6-Phenyl $110 \AA$ column (Phenomenex, Torrance, CA, USA). $30 \mu \mathrm{L}$ crudely purified metabolites were injected on the column using a flow of $5.000 \mathrm{~mL} / \mathrm{min}$ with a linear water-acetonitrile gradient, where both were buffered with $50 \mathrm{ppm}$ trifluoroacetic acid. The gradient started at $0 \%$ acetonitrile and reached $60 \%$ acetonitrile in $10 \mathrm{~min}$, which were held for $1 \mathrm{~min}$ before reverting to $0 \%$ acetonitrile.

A

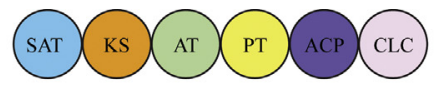

B

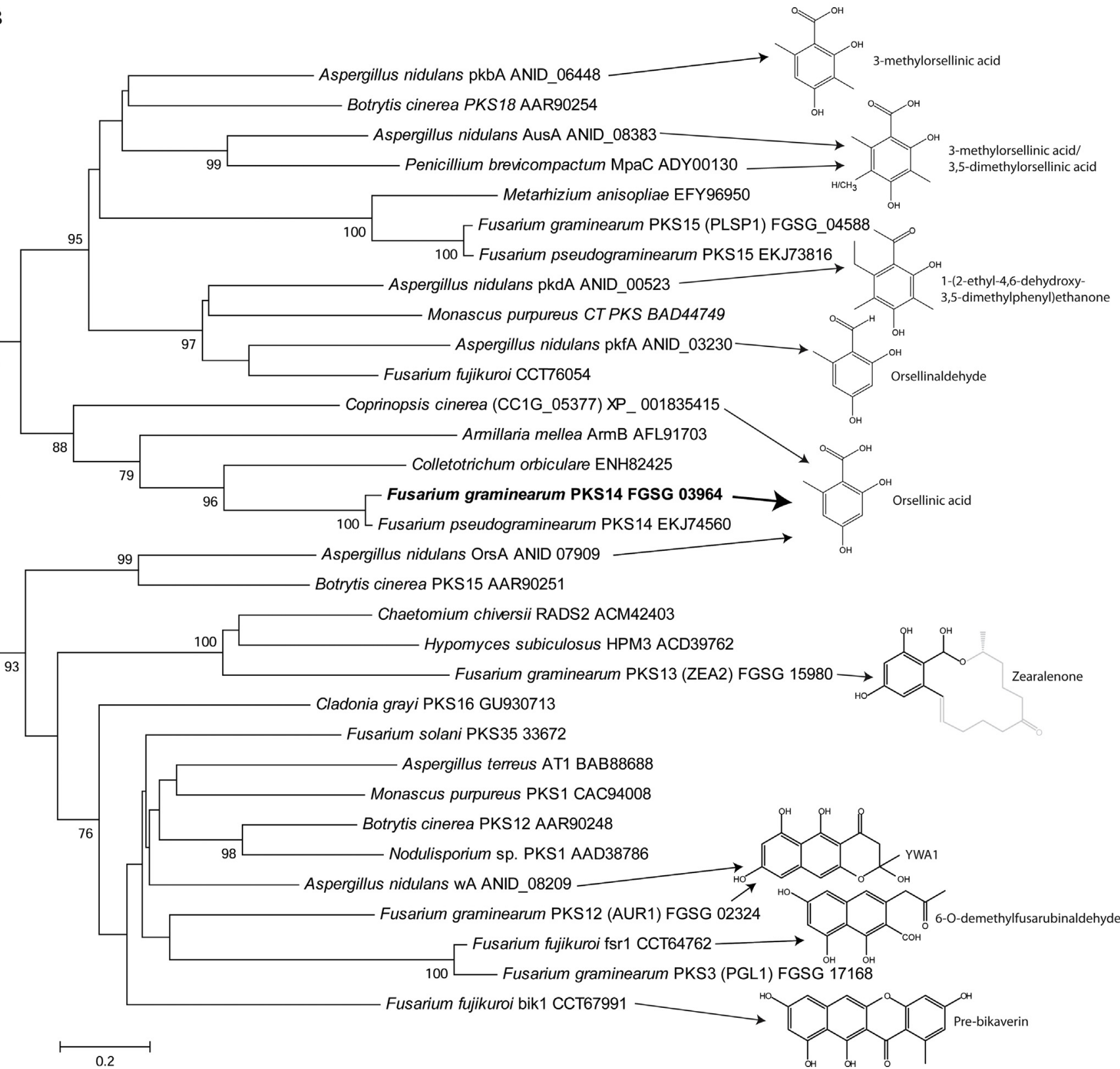

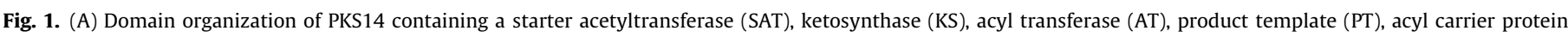

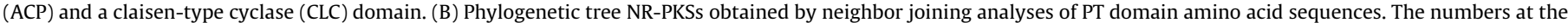

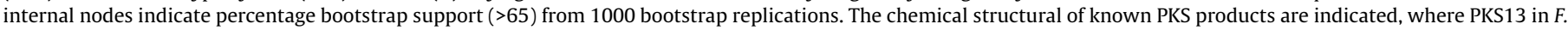
graminearum hypothetically produces the highlighted part of zearalenone (Zhou et al., 2008). 
A well resolved peak eluting at $5.4 \mathrm{~min}$, corresponding to the retention time of authentic orcinol, was collected.

\subsection{Structural verification}

The structure was verified using nuclear magnetic resonance spectroscopy (NMR) on a Bruker AVIII-600 spectrometer (Bruker, Karlsruhe, Germany). Approximately $5 \mu \mathrm{g}$ of the compound was dissolved in $600 \mu \mathrm{L} \mathrm{CDCl}_{3}$ and analyzed with ${ }^{1} \mathrm{H},{ }^{13} \mathrm{C}$ and $2 \mathrm{D}-\left[{ }^{1} \mathrm{H}-{ }^{13} \mathrm{C}\right]-\mathrm{HSQC}$ at $291 \mathrm{~K}$. Spectra were recorded and analyzed with TopSpin 3.2 (Bruker). All chemical shifts are relative to internal tetramethylsilane (TMS).

\section{Results and discussion}

\subsection{Phylogenetic analyses of PKS14}

PKS14 contains a starter acetyltransferase (SAT), ketosynthase (KS), acyl transferase (AT), product template (PT), acyl carrier protein (ACP) and a claisen-type cyclase (CLC) domain (Fig. 1A). To gain insight into the chemical product of PKS14, we performed a phylogenetic analysis of the predicted amino acids corresponding to the PT and KS domain from PKS14 and 31 other NR-PKSs (Fig. 1B and Supplementary Fig. 1). The PKSs include five from A. nidulans shown to produce orsellinic acid or derivatives (Ahuja et al., 2012), a PKS from Cladonia grayi hypothesized to be responsible for production of orsellinic acid in route to the synthesis of the orcinol depsidone grayanic acid (Armaleo et al., 2011), and a PKS from Coprinopsis cinerea shown in Saccharomyces cerevisiae to produce orsellinic acid (Ishiuchi et al., 2012). The resulting genealogy resolved the 32 PKSs into three major clades each with $100 \%$ bootstrap support. PKS14 was present in a clade with the orsellinic acid synthase from $C$. cinerea and a PKS (armB) from the orsellinic acid producer Armillaria mellea (100\% bootstrap support in analyses with KS domains, $88 \%$ in analyses with PT domains). Compared to the $A$. nidulans orsellinic acid synthase (anid_07909), the C. cinerea synthase lacks the SAT domain and the second copy of ACP. As observed by Lackner et al. (2012), the two synthases do not cluster together in KS based phylogenetic analyses. This suggests that PKS14 from $F$. graminearum is a member of orsellinic acid synthases, which are polyphyletic and can have variable domain structures.

\subsection{Prediction of the PKS14 gene cluster}

To examine the extent of the putative PKS14 gene cluster, the transcription of PKS14 and eight upstream and downstream genes were analyzed (Fig. 2A). The expression profile of PKS14 clustered together with a decarboxylase (FGSG_03965) as well as six other genes. These six genes are predicted to encode two proteins with unknown functions (FGSG_03958 and FGSG_16177), an amidase/ hydrolase (FGSG_03963), a hydrophobin protein (FGSG_03960), a cytochrome P450 monooxygenase (FGSG_12342) and a carboxypeptidase (FGSG_03967) (Fig. 2B). These co-regulated genes are

\section{A}

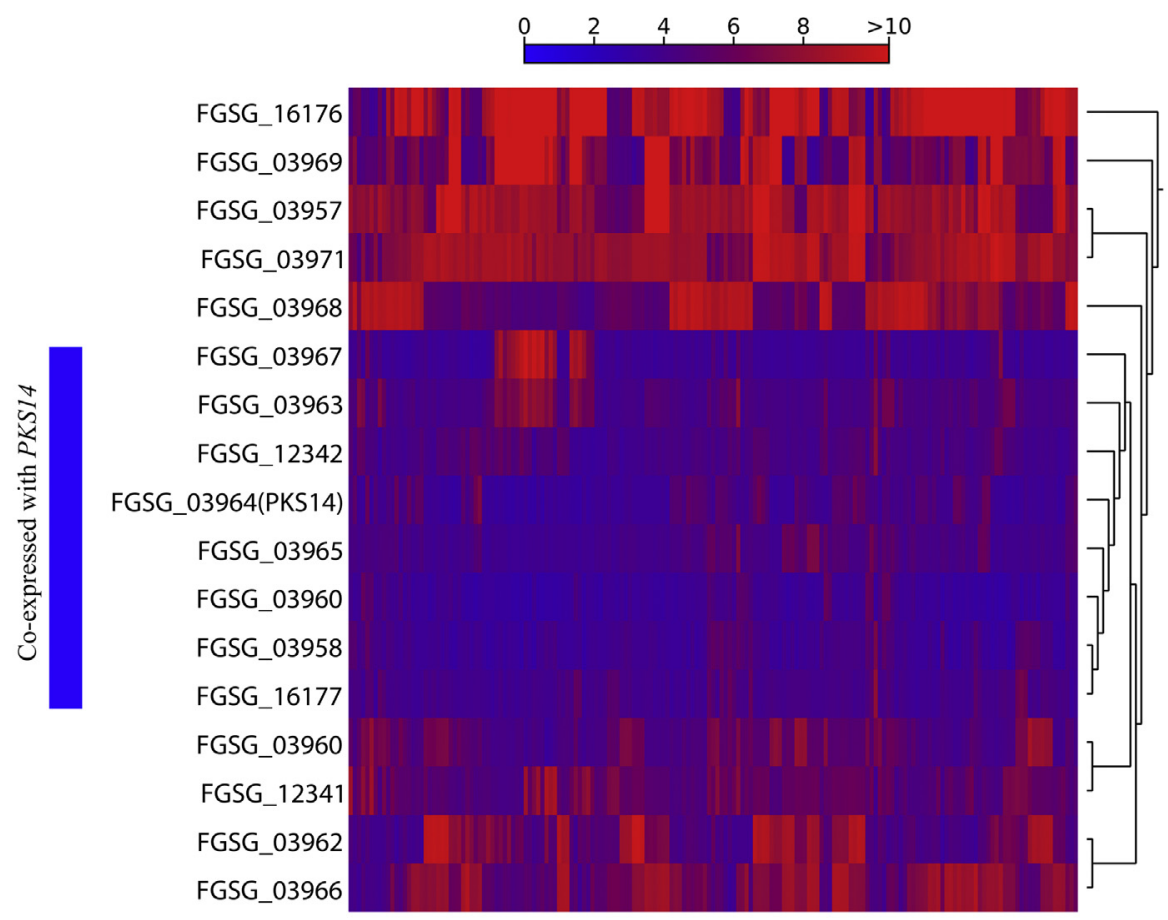

B

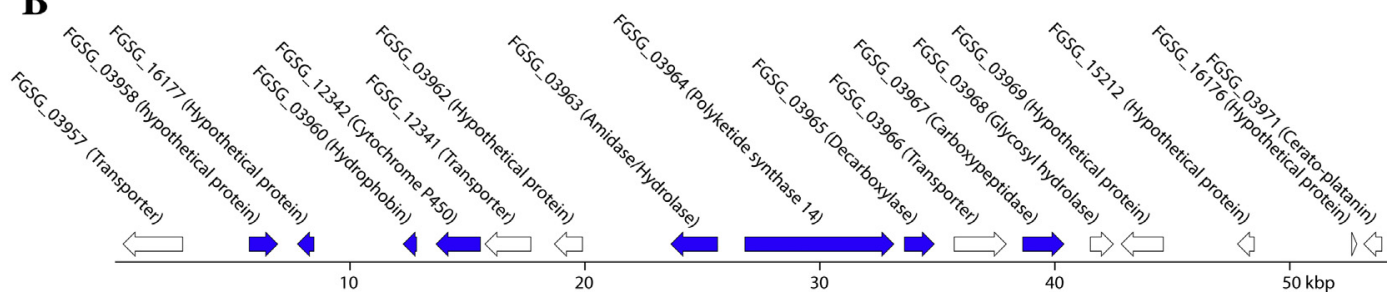

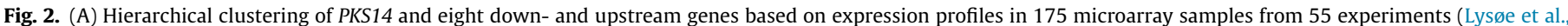

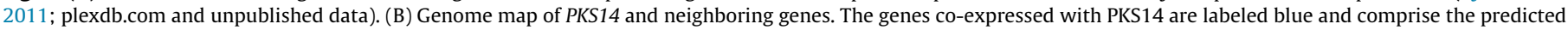
cluster. The predicted function of the genes was determined through the NCBI Conserved Domain Database (Marchler-Bauer et al., 2011). 

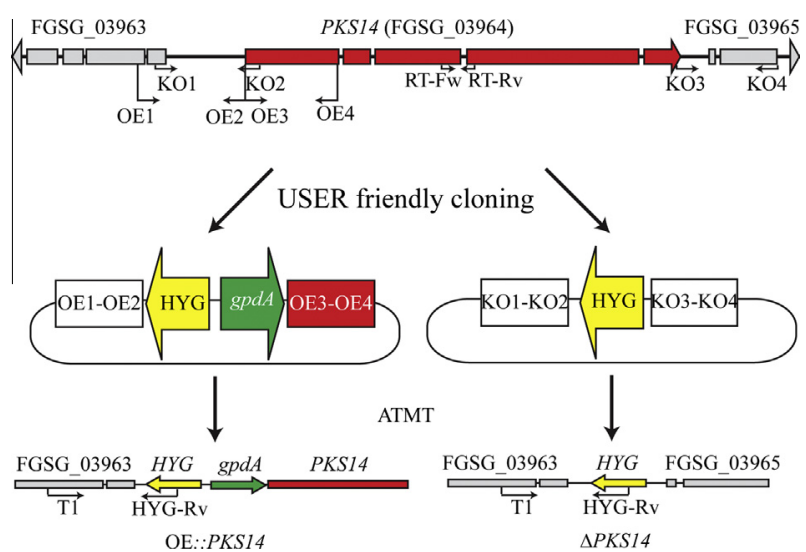

Fig. 3. Generation of overexpressing and deletion mutants of PKS14. Overexpressing mutants are obtained by cloning amplified regions OE1-OE2 and OE3-OE4 into a p-HU2E vector containing the HYG gene (hygromycin resistance) and the gpdA constitutive promoter. Deletion mutants are obtained by cloning amplified regions $\mathrm{KO} 1-\mathrm{KO} 2$ and $\mathrm{KO} 3-\mathrm{KO} 4$ into p-HU2. Both vectors were introduced into $F$. graminearum by $A$. tumefaciens mediated transformation and inserted into the genome by homologous recombination.

proposed to make up the PKS14 gene cluster. The remaining three genes which are located inside the cluster, but are not co-regulated, are predicted to encode a protein with unknown function and two putative transporters. The expression profile of these three genes clustered together to form a separate subclade. The remaining genes surrounding the predicted cluster are more abundantly expressed under most of the conditions examined and are not considered part of the predicted PKS14 cluster.

\subsection{Generation and characterization of PKS14 deletion and overexpression mutants}

Deletion mutants of PKS14 were successfully generated by gene replacements using Agrobacterium mediated transformation (Fig. 3). The constitutive A. nidulans promoter gpdA was introduced in front of PKS14 for in locus overexpression of the gene. Correct integration of the transformation vectors into the genome was verified with PCR and single transformation events confirmed by Southern blot analysis (Supplementary Fig. 2). Two overexpression mutants and one deletion mutant were selected for further studies.

These mutant strains were grown on rice medium (RM), potato dextrose agar (PDA) and yeast extract sucrose (YES) medium to examine whether deletion of PKS14 had an inhibiting effect on growth rate as reported by Gaffoor et al. (2005). In our experiments, we did not observe any significant differences in the radial growth of the strains after five days (Fig. 4A). After seven days of growth the mycelium had covered the growth medium and exhibited similar morphology to that of the wild type (Fig. 4B).

Gaffoor et al. (2005) have previously shown that growth on rice medium induces expression of PKS14. To examine changes in the transcription level of the PKS14 cluster in the mutant strains, the mutants and wild type were, grown on this medium and RNA was extracted. The RT-PCR showed that PKS14 was transcribed by the OE::PKS14 mutants and the wild type strain, while no transcript was observed in the $\triangle P K S 14$ mutant (Fig. 4C). The remaining genes were all expressed in the different strains, although there was a tendency that the intensity was weaker for some of the genes in the $\triangle$ PKS14 mutant compared to the wild type.

\subsection{Metabolite analyses}

To detect changes in the production of secondary metabolites, extracts of strains grown on RM medium for two weeks were analyzed. The extracts were initially screened by reverse phase HPLCUV, where we observed an early eluting peak from a compound, which was present in OE::PKS14 mutants, but not in the wild type or the $\triangle P K S 14$ mutant. The samples were subsequently analyzed by UHPLC-HRMS, where the compound produced by the OE::PKS14 mutants was detected as a very poorly ionizing peak with $[\mathrm{M}+\mathrm{H}]^{+}$ at a $m / z 125.0587$ (and no ionization in $\mathrm{ESI}^{-}$) corresponding to an elemental composition of $\mathrm{C}_{7} \mathrm{H}_{8} \mathrm{O}_{2}$ (calculated $[\mathrm{M}+\mathrm{H}]^{+}$125.0602) (Fig. 5A). The compound was identified as orcinol by comparing the elution time, accurate mass and fragmentation pattern to a commercial reference standard. Another fungal metabolite with this elemental composition, 3-methylbenzene-1,2-diol, was acquired (Sigma-Aldrich) and co-analyzed showing that this positional isomers was easily separated from orcinol.

Orcinol can be produced by decarboxylation of orsellinic acid (Pettersson, 1965; Mosbach and Ehrensvärd, 1966), and we were also able to detect this compound by UHPLC-HRMS in extracts from the two OE::PKS14 mutants (Fig. 5B). The identity of orsellinic acid was verified by comparing retention time, HRMS using extracted ion-chromatograms $\mathrm{m} / z \pm 0.01$ and by measuring the accurate mass of $[\mathrm{M}+\mathrm{H}]+$ as 169.0492 (Calculated 169.0495, deviation $-2.0 \mathrm{ppm}$ ). To verify the identity of orcinol, the compound was isolated by preparative HPLC and analyzed by NMR. The levels of orsellinic acid produced by the mutant strains were too low to obtain satisfactory amounts for NMR validation. The ${ }^{1} \mathrm{H}$ and ${ }^{13} \mathrm{C}$ NMR spectra of the isolated orcinol identified six $\mathrm{H}$-atoms and at least five C-atoms (Table 1, Supplementary Fig. 3). The measured chemical shifts matched those of orcinol reported by Richter et al. (2011), although the chemical shifts for the two hydroxyl groups were not obtained, due to rapid exchange.
A

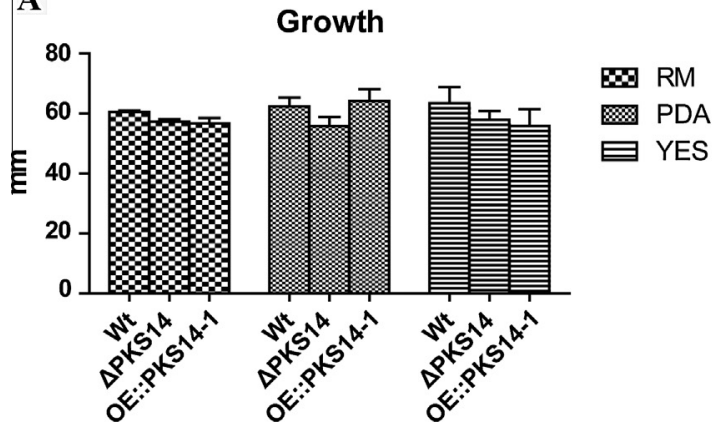

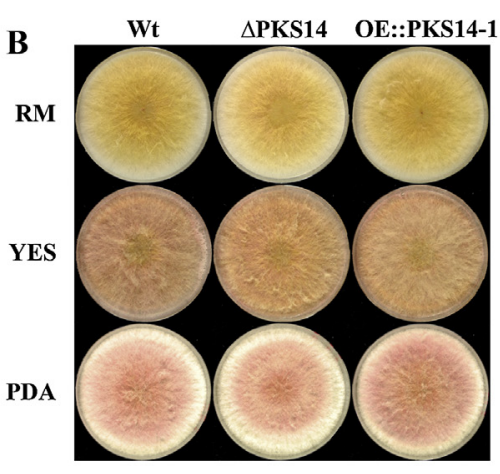

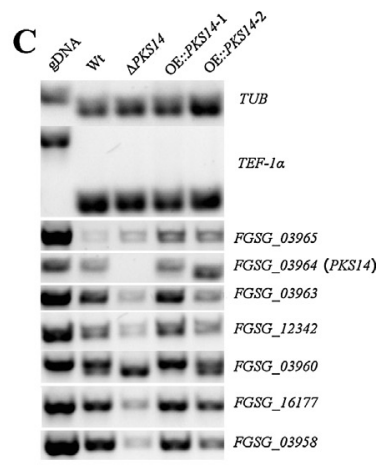

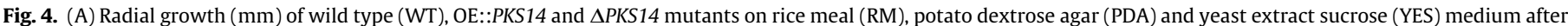

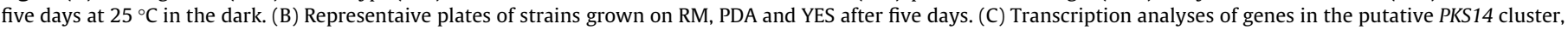
translation elongation factor $1 \alpha$ (TEF-1 $\alpha$ ) and $\beta$-tubulin ( $\beta$-tub) in the WT, OE::PKS14 and $\triangle P K S 14$ mutants. 
$\mathbf{A}$
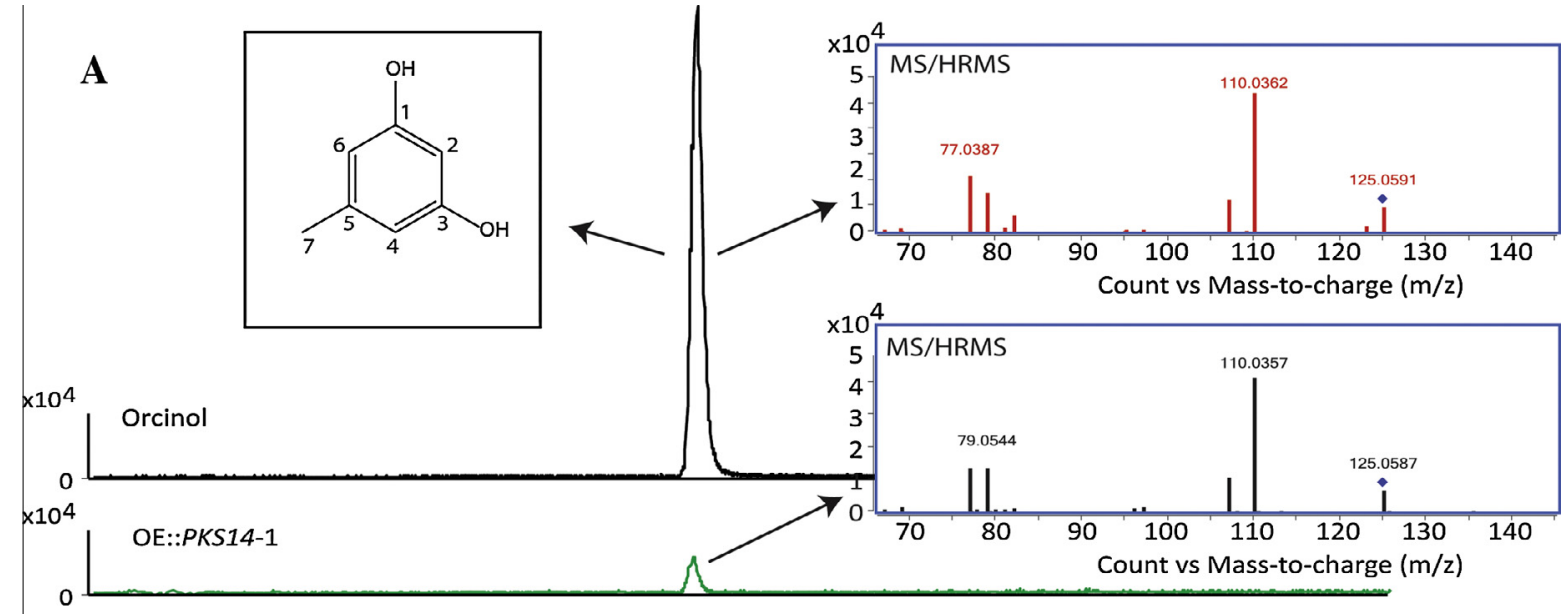

$\times 10^{4}$

\section{OE::PKS14-2}

0
$\times 104$

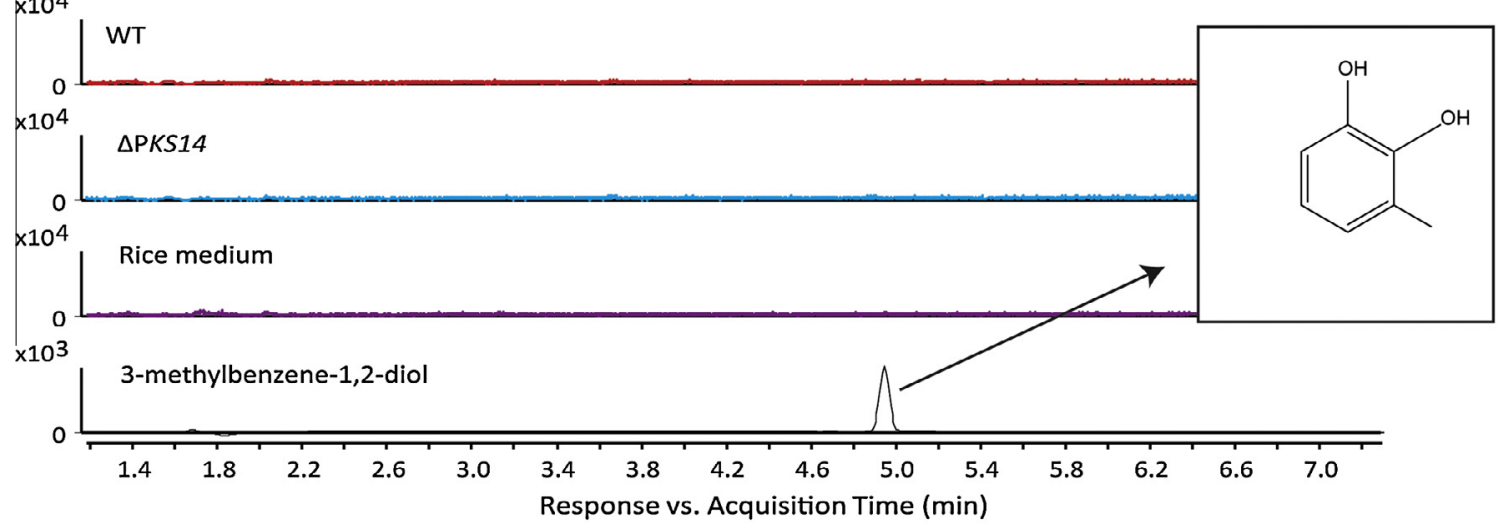

B
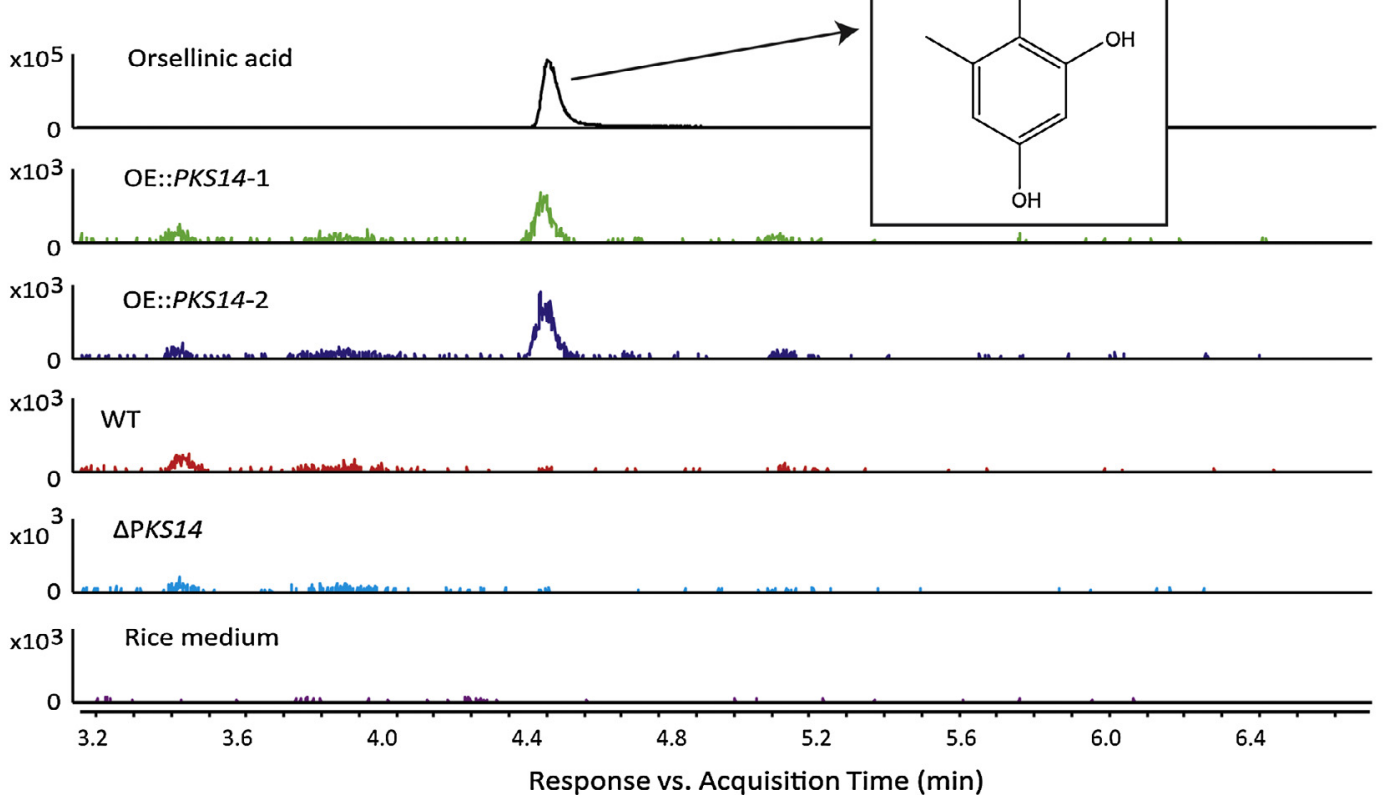

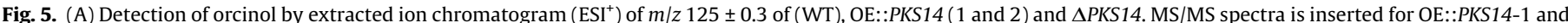

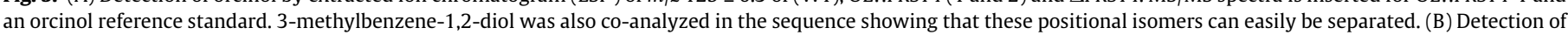
orsellinic acid in the stains by ion chromatogram $\left(\mathrm{ESI}^{+}\right)$of $m / z 169.0495 \pm 0.01$ compared to a reference standard. 
Table 1

Chemical shifts of the isolated orcinol in $\mathrm{CDCl}_{3}$ at $291 \mathrm{~K}$.

\begin{tabular}{lll}
\hline Atom & ${ }^{13} \mathrm{C}^{\mathrm{a}}$ & ${ }^{1} \mathrm{H}^{\mathrm{a}}$ \\
\hline 1,3 & $141.0 / 156.6$ & - \\
2 & 99.9 & 6.16 \\
4,6 & 108.7 & 6.24 \\
5 & $141.0 / 156.6$ & - \\
7 & 21.4 & 2.24
\end{tabular}

a Chemical shifts are relative to internal tetramethylsilane (TMS).

Orsellinic acid represents the archetypal phenolic polyketide because it is the simplest acetate-derived aromatic metabolite and is a precursor in numerous biosynthetic pathways (Schroeckh et al., 2009). It is interesting that orsellinic acid can be produced by synthases which do not have same domain architecture and release mechanism. The orsellinic acid synthase (orsA) in $A$. nidulans has a thioesterase (TE) release domain, whereas PKS14 has a CLC domain. Furthermore, PKS14 and the orsellinic acid synthase from $C$. cinerea (CC1G_05377) have one ACP domain, whereas orsA has two.

Orcinol has been isolated from numerous lichen fungi (Robiquet, 1829) and can be synthesized by decarboxylation of orsellinic acid in Umbilicaria papulosa and Gliocladium roseum (Pettersson, 1965; Mosbach and Ehrensvärd, 1966). Umbilicaria spp. can furthermore produce numerous orsellinic acid-derived depsides and tridepsides (Narui et al., 1998), whereas G. roseum produce gliorosein, derived from orsellinic acid (Packter and Steward, 1967). These compounds were however not detected in the PKS14 overexpressing mutants. The putative decarboxylase (FGSG_03965), which is present directly upstream of PKS14, is likely to catalyze the step from orsellinic acid to orcinol. This hypothesis is supported by the microarray data and RT-PCR results, which showed that the decarboxylase is co-regulated with PKS14. The decarboxylases from $U$. papulosa and G. roseum have not been sequenced and it therefore unknown whether the putative $F$. graminearum orsellinic acid decarboxylase are homologues. The orsellinic acid gene cluster in A. nidulans contains a putative decarboxylase (AN7911) and tyrosinase/polyphenol oxidase (AN7912) and in a wild type strain the cluster is responsible for production of lecanoric acid F-9775A and B (Schroeckh et al., 2009; Sanchez et al., 2010). Deletion of the tyrosinase and decarboxylase in $A$. nidulans resulted in accumulation of gerfelin and diorcinol, which was not detected in the wild type strain (Sanchez et al., 2010). Whether the decarboxylase is involved in biosynthesis of diorcinol is still hypothetical.

As our expression analyses suggests that PKS14 belongs to a gene cluster, orcinol might not be the true end product of the biosynthetic pathway in F. graminearum as some of the putative cluster genes can potentially perform additional modifications to orcinol. Furthermore, orcinol is a substructure found in many fungal compounds, but as we only detected orcinol in the extracts from the overexpression mutants further investigations are needed to elucidate the full biosynthetic pathway of the PKS14 gene cluster.

\section{Conclusions}

In the present study, we show that $F$. graminearum is capable of producing orsellinic acid and orcinol when PKS14 was overexpressed. Although the wild type strain does not produce the compounds in detectable amounts under the tested conditions it is possible they may be found during infection of wheat, where transcriptome analyses have shown that the gene is expressed (Lysøe et al., 2011). The entire biosynthetic pathway of PKS14 has probably not been fully revealed in the present study and further studies are needed to identify the fate and function of orsellinic acid and orcinol in F. graminearum.

\section{Acknowledgments}

The study was supported by grants from The Danish Research Council, Technology and Production (12-132415 and 10-100105). The authors are grateful to Agilent Technologies for donation of the UHPLC-qTOF instrument under the Thought leader program. The NMR laboratory at Aalborg University is supported by the Obel, SparNord and Carlsberg Foundations.

\section{Appendix A. Supplementary material}

Supplementary data associated with this article can be found, in the online version, at http://dx.doi.org/10.1016/j.fgb.2014.06.008.

\section{References}

Ahuja, M., Chiang, Y., Chang, S., Praseuth, M.B., Entwistle, R., Sanchez, J.F., Lo, H., Yeh, H., Oakley, B.R., Wang, C.C.C., 2012. Illuminating the diversity of aromatic polyketide synthases in Aspergillus nidulans. J. Am. Chem. Soc. 134, 8212-8221.

Armaleo, D., Sun, X., Culberson, C., 2011. Insights from the first putative biosynthetic gene cluster for a lichen depside and depsidone. Mycologia 103 $741-754$.

Bergmann, S., Schumann, J., Scherlach, K., Lange, C., Brakhage, A.A., Hertweck, C. 2007. Genomics-driven discovery of PKS-NRPS hybrid metabolites from Aspergillus nidulans. Nat. Chem. Biol. 3, 213-217.

Bode, H.B., Bethe, B., Hofs, R., Zeeck, A., 2002. Big effects from small changes: possible ways to explore nature's chemical diversity. ChemBioChem 3, 619627.

Burlakoti, R.R., Ali, S., Secor, G.A., Neate, S.M., McMullen, M.P., Adhikari, T.B., 2008 Genetic relationships among populations of Gibberella zeae from barley, wheat potato, and sugar beet in the upper Midwest of the United States. Phytopathology 98, 969-976.

Droce, A., Sorensen, J.L., Giese, H., Sondergaard, T.E., 2013. Glass bead cultivation of fungi: combining the best of liquid and agar media. J. Microbiol. Methods 94 343-346.

Fisch, K.M., Gillaspy, A.F., Gipson, M., Henrikson, J.C., Hoover, A.R., Jackson, L., Najar, F.Z., Wagele, H., Cichewicz, R.H., 2009. Chemical induction of silent biosynthetic pathway transcription in Aspergillus niger. J. Ind. Microbiol. Biotechnol. 36, 1199-1213.

Frandsen, R.J., Nielsen, N.J., Maolanon, N., Sorensen, J.C., Olsson, S., Nielsen, J., Giese, H., 2006. The biosynthetic pathway for aurofusarin in Fusarium graminearum reveals a close link between the naphthoquinones and naphthopyrones. Mol Microbiol. 61, 1069-1080.

Frandsen, R.J., Andersson, J.A., Kristensen, M.B., Giese, H., 2008. Efficient four fragment cloning for the construction of vectors for targeted gene replacement in filamentous fungi. BMC Mol. Biol. 9, 1-11.

Gaffoor, I., Trail, F., 2006. Characterization of two polyketide synthase genes involved in zearalenone biosynthesis in Gibberella zeae. Appl. Environ. Microbiol. 72, 1793-1799.

Gaffoor, I., Brown, D.W., Plattner, R., Proctor, R.H., Qi, W., Trail, F., 2005. Functional analysis of the polyketide synthase genes in the filamentous fungus Gibberella zeae (anamorph Fusarium graminearum). Eukaryot. Cell 4, 1926-1933.

Hansen, F.T., Droce, A., Sørensen, J.L., Fojan, P., Giese, H., Sondergaard, T.E., 2012a. Overexpression of NRPS4 leads to increased surface hydrophobicity in Fusarium graminearum. Fungal Biol. 116, 855-862.

Hansen, F.T., Sørensen, J.L., Giese, H., Sondergaard, T.E., Frandsen, R.J., 2012b. Quick guide to polyketide synthase and nonribosomal synthetase genes in Fusarium. Int. J. Food Microbiol. 155, 128-136.

Ishiuchi, K., Nakazawa, T., Ookuma, T., Sugimoto, S., Sato, M., Tsunematsu, Y. Ishikawa, N., Noguchi, H., Hotta, K., Moriya, H., Watanabe, K., 2012. Establishing a new methodology for genome mining and biosynthesis of polyketides and peptides through yeast molecular genetics. ChemBioChem 13, 846-854.

Keller, N.P., Hohn, T.M., 1997. Metabolic pathway gene clusters in filamentous fungi. Fungal Genet. Biol. 21, 17-29.

Kim, Y.T., Lee, Y.R., Jin, J., Han, K.H., Kim, H., Kim, J.C., Lee, T., Yun, S.H., Lee, Y.W., 2005. Two different polyketide synthase genes are required for synthesis of zearalenone in Gibberella zeae. Mol Microbiol. 58, 1102-1113.

Kroken, S., Glass, N.L., Taylor, J.W., Yoder, O.C., Turgeon, B.G., 2003. Phylogenomic analysis of type I polyketide synthase genes in pathogenic and saprobic ascomycetes. Proc. Natl. Acad. Sci. U.S.A. 100, 15670-15675.

Lackner, G., Misiek, M., Braesel, J., Hoffmeister, D., 2012. Genome mining reveals the evolutionary origin and biosynthetic potential of basidiomycete polyketide synthases. Fungal Genet. Biol. 49, 996-1003.

Li, Y., Xu, W., Tang, Y., 2010. Classification, prediction, and verification of the regioselectivity of fungal polyketide synthase product template domains. J. Biol. Chem. 285, 22764-22773. 
Lysøe, E., Seong, K.Y., Kistler, H.C., 2011. The transcriptome of Fusarium graminearum during the infection of wheat. Mol, Plant Microbe Interact. 24. $995-1000$.

Malz, S., Grell, M.N., Thrane, C., Maier, F.J., Rosager, P., Felk, A., Albertsen, K.S., Salomon, S., Bohn, L., Schäfer, W., Giese, H., 2005. Identification of a gene cluster responsible for the biosynthesis of aurofusarin in the Fusarium graminearum species complex. Fungal Genet. Biol. 42, 420-433.

Marchler-Bauer, A., Lu, S., Anderson, J.B., Chitsaz, F., Derbyshire, M.K., DeWeeseScott, C., Fong, J.H., Geer, L.Y., Geer, R.C., Gonzales, N.R., Gwadz, M., Hurwitz, D.I., Jackson, J.D., Ke, Z., Lanczycki, C.J., Lu, F., Marchler, G.H., Mullokandov, M., Omelchenko, M.V., Robertson, C.L., Song, J.S., Thanki, N., Yamashita, R.A., Zhang, D., Zhang, N., Zheng, C., Bryant, S.H., 2011. CDD: a conserved domain database for the functional annotation of proteins. Nucleic Acids Res. 39, D225-D229.

Mosbach, K., Ehrensvärd, U., 1966. Studies on lichen enzymes Part I. Preparation and properties of a depside hydrolysing esterase and of orsellinic acid decarboxylase. Biochem. Biophys. Res. Commun. 22, 145-150.

Narui, T., Sawada, K., Takatsuki, S., Okuyama, T., Culberson, C.F., Culberson, W.L. Shibata, S., 1998. NMR assignments of depsides and tridepsides of the lichen family Umbilicariaceae. Phytochemistry 48, 815-822.

Nielsen, K.F., Månsson, M., Rank, C., Frisvad, J.C., Larsen, T.O., 2011. Dereplication of microbial natural products by LC-DAD-TOFMS. J. Nat. Prod. 74, 2338-2348.

Nielsen, M.T., Nielsen, J.B., Anyaogu, D.C., Holm, D.K., Nielsen, K.F., Larsen, T.O., Mortensen, U.H., 2013. Heterologous reconstitution of the intact geodin gene cluster in Aspergillus nidulans through a simple and versatile PCR based approach. PLoS One 8, 1-10.

Packter, N.M., Steward, M.W., 1967. Studies on the biosynthesis of phenols in fung - biosynthesis of 3,4-dimethoxy-6-methyltoluquinol and gliorosein in
Gliocladium into the acetogenin (polyketide) gliorosein in Gliocladium roseum I.M.I. 93065. Biochem. J. 102, 122-132.

Pettersson, G., 1965. An orsellinic acid decarboxylase isolated from Gliocladium roseum. Acta Chem. Scand. 19, 2013-2021.

Richter, H., Beckendorf, S., Mancheño, O.G., 2011. Modifiable sulfur tethers as directing groups for aromatic $\mathrm{C}-\mathrm{H}$ acetoxylation reactions. Adv. Synth. Catal. 353, 295-302.

Robiquet, P., 1829. Essai Analytique des Lichens de l'Orseille. Ann. Chim. Phys. 42, $236-257$.

Sanchez, J.F., Chiang, Y., Szewczyk, E., Davidson, A.D., Ahuja, M., Elizabeth Oakley, C., Woo Bok, J., Keller, N., Oakley, B.R., Wang, C.C.C., 2010. Molecular genetic analysis of the orsellinic acid/F9775 gene cluster of Aspergillus nidulans. Mol. BioSyst. 6, 587-593.

Schroeckh, V., Scherlach, K., Nutzmann, H.W., Shelest, E., Schmidt-Heck, W., Schuemann, J., Martin, K., Hertweck, C., Brakhage, A.A., 2009. Intimate bacterial-fungal interaction triggers biosynthesis of archetypal polyketides in Aspergillus nidulans. Proc. Natl. Acad. Sci. U.S.A. 106, 14558-14563.

Smedsgaard, J., 1997. Micro-scale extraction procedure for standardized screening of fungal metabolite production in cultures. J. Chromatogr. A 760, 264-270.

Sørensen, J.L., Hansen, F.T., Sondergaard, T.E., Staerk, D., Lee, T.V., Wimmer, R., Klitgaard, L.G., Purup, S., Giese, H., Frandsen, R.J., 2012. Production of novel fusarielins by ectopic activation of the polyketide synthase 9 cluster in Fusarium graminearum. Environ. Microbiol. 14, 1159-1170.

Tamura, K., Peterson, D., Peterson, N., Stecher, G., Nei, M., Kumar, S., 2011. MEGA5: molecular evolutionary genetics analysis using maximum likelihood, evolutionary distance, and maximum parsimony methods. Mol. Biol. Evol. 28, $2731-2739$. 